\title{
Results of the Treatment Vascular Anomalies with the 5 ALA Induced Photodynamic Therapy
}

\section{Sadykov Rasul Rustamovich*}

Maxillofacial Surgery Department, Ernst Moritz Arndt University of Greifswald, Germany

\begin{abstract}
This study reports the role of photodynamic therapy in the management of vascular anomalies. A 3-year (2009-2012) retrospective study on the challenges and outcome of two hundred forty five patients with vascular anomalies referred for surgical management was undertaken at the Tashkent Medical Academy, Uzbekistan. After multidisciplinary discussion, all patients underwent photodynamic therapy under general anesthesia, with 5-ALA as the photosensitising agent. In a time of treatment by eighty five out of one hundred and eight patients, who presented with long-term pain, reported improvement after treatment. Also, 43/46 reported significant reduction of bleeding related to their vascular anomaly. Improvement of swelling was reported by $189 / 199$ patients; while reduction of infection episodes was evident in 61/63 patients and 176/205 reported reduction in the disfigurement caused by their pathology. Clinical assessment showed that more than half of the patients had 'good response' to the treatment. Significant clinical response was reported by $148(60,4 \%)$ patients, moderate result by $70(28,6 \%)$. Radiological and ultrasound assessment comparing imaging 6-week post-laser and PDT to the baseline showed moderate response in $78(31,8 \%)$ patients and significant response in $122(49,8 \%)$ patients.
\end{abstract}

Keywords: Hemangioma; Congenital and infantile; Malformations; Laser; Surgery; Photodynamic therapy; 5-ALA

\section{Introduction}

Vascular anomalies are congenital anomalies of vascular development and cause various degrees of soft tissue abnormalities. These anomalies tend to occur most commonly in the head and neck and affect approximately 1 in 22 children [1,2]. The most recent classification of vascular anomalies ISSVA includes two main categories: vascular tumours (hemangiomas) and vascular malformations. Vascular tumours include 'infantile' and 'congenital' type haemangiomas; the former being the most common vascular tumour as well as vascular anomaly. Other types include pyogenic granuloma, tufted angiomas and haemangioendotheliomas, angiosarcomas [3-5].

Haemangiomas are more common in females and $80 \%$ occur in the head and neck [3,6]. Haemangiomas usually present isolated, multifocal or segmental result from endothelial cell hyperplasia. Infantile haemangiomas develop shortly after birth and follow the expected course of proliferation and prolonged involution. They are one of the most vascular tumour. The 'rarer' congenital haemangioma do not follow the same growth pattern but it does present at birth as the result of endothelial cell hyperplasia; and may rapidly involute or never involute. The causes of the aberrant and focal proliferation of endothelial cells in these lesions remain unclear $[1,7]$.

Venous malformations are usually slow-flow vascular malformations composed of ecstatic venous channels that continue to grow throughout the patient's life. They commonly occur in the head and neck area, with a predilection for the oral cavity, airway and muscle groups. These lesions have unpredicted growth behavior when it comes to how much growth will occur or where; and many lesions expand well beyond their initial clinical boundaries and actual invasion of surrounding tissue may be occurring. These lesions are frequently symptomatic, depending on the locations involved and are usually obvious at birth. They fill with dependency and are compressible with variable color depending on the depth of involvement. Symptoms can be related to clot formation either from trauma or venous stasis and patients usually present with pain and swelling $[8,9]$.
The various therapeutic options are available for vascular anomalies as well as the recent advances in psychosocial aspects of care, interventional radiology, laser and pharmacological therapy $[10,11]$.

\section{Photodynamic therapy}

Photodynamic therapy (PDT) is a developing technology, used in the treatment of advanced tumour. The main application for PDT is to target tumour of the head and neck, gastrointestinal tract, pulmonary malignancies and skin pathologies. One advantageous feature of photodynamic therapy is that it does not cause damage to nerves (cold photochemical reaction); this has lead to its use in the treatment of benign neoplasm's $[12,13]$.

The effects of PDT are a result of the interaction between a photosensitiser, oxygen and light. PDT may be provided under local, regional or general anesthesia and it may be delivered by surface illumination or interstitial application, via inserted optical fibers. The photosensitiser, administered whether locally or systemically, is selectively taken up and retained by tissues with a high vascular flow rate. Activation by light of a specific wavelength results in the production of oxygen free radicals or singlet oxygen which leads to apoptosis and/ or direct cell death, as well as vascular shut down and subsequent inflammatory/immunological response $[4,14]$. Traditionally, light is delivered to the target tissue by surface illumination, whereby an optic probe is held over the target tissue (distance $5 \mathrm{~cm}$ ). It can penetrate up to a depth of $1 \mathrm{~cm}$, when used with 5-ALA due to its wavelength and

*Corresponding author: Sadykov Rasul Rustamovich, Maxillofacial Surgery Department, Ernst Moritz Arndt University of Greifswald, Germany, Tel: +491747076690; E-mail: srrdoktor@yahoo.com

Received August 23, 2013; Accepted December 23, 2013; Published December 25, 2013

Citation: Rustamovich SR (2013) Results of the Treatment Vascular Anomalies with the 5 ALA Induced Photodynamic Therapy. Trop Med Surg 1: 157. doi:10.4172/2329-9088.1000157

Copyright: @ 2013 Rustamovich SR. This is an open-access article distributed under the terms of the Creative Commons Attribution License, which permits unrestricted use, distribution, and reproduction in any medium, provided the original author and source are credited. 
Citation: Rustamovich SR (2013) Results of the Treatment Vascular Anomalies with the 5 ALA Induced Photodynamic Therapy. Trop Med Surg 1: 157. doi:10.4172/2329-9088.1000157


Figure 1: Capillary malformation upper eyebrow, lips subjected to PDT. Clinical evaluation reported 'good response' to the therapy. Radiological response was 'significant'.
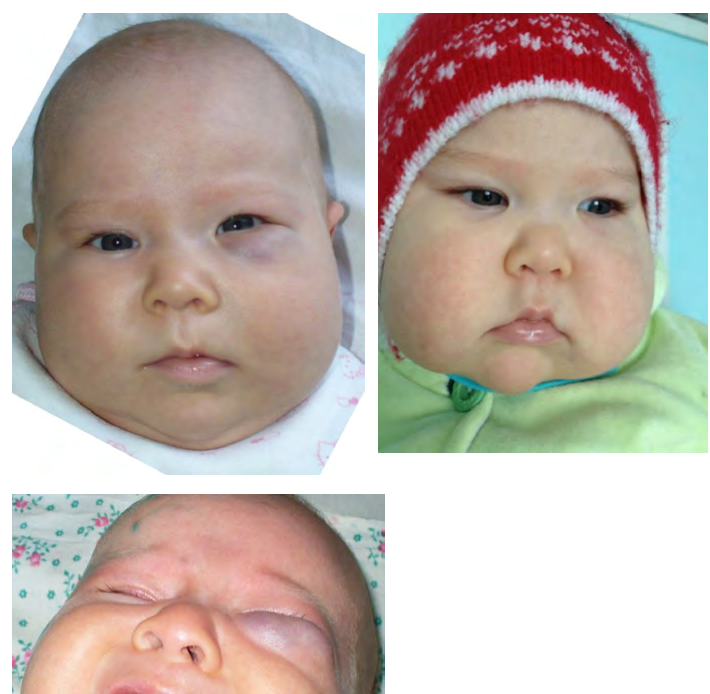

Figure 2: Venous malformation lower eyelid subjected to PDT. Clinical evaluation was 'good response'.

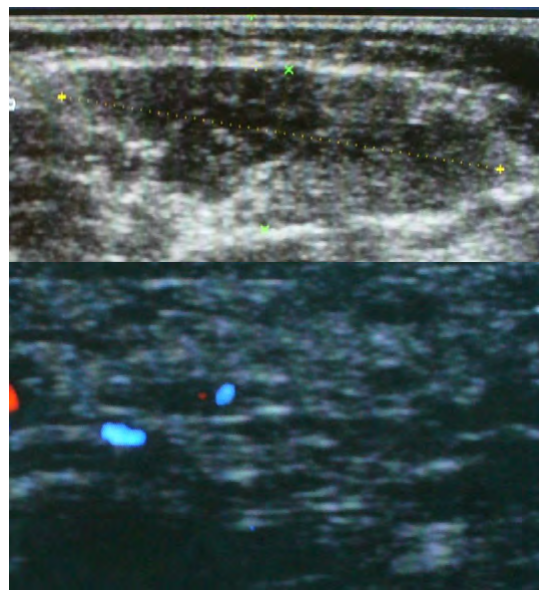

Figure 3: Radiological evaluation reported 'good response' to the therapy.

not properties of the photosensitiser. Interstitial PDT followed by the insertion of thin optical fibers into the needles, allows light maximal tissue illumination [15]. The application of iPDT in combination with preoperative imaging, including computer tomography $(\mathrm{CT})$, magnetic resonance imaging (MRI) and Ultrasound images has been shown to improve the accuracy of the technique. In this study we present the outcome following the management of vascular anomalies using photodynamic therapy. Patients' reports on quality of life with clinical and radiological evaluation were the main parameters used to assess the outcome (Figure 3).

\section{Material and Methods}

In this clinical study, 245 patients (54 males and 191 females) were referred to the general and maxilla-facial surgery departments, Tashkent medical academy, Uzbekistan (between 2009 and 2012) for treatment of a range of vascular anomalies. The mean age was $1,2 \pm$ 0,4 years (Min 0.1, Max 34) with 89, 8\% of the cohort being Central Asian, $7,7 \%$ Europeans, 2,4\% different. The most common primary sites were the mid face, lower face, upper face, oral cavity and neck. Vascular anomalies included vascular tumour (infantile haemangiomas in 74 patients and congenital haemangiomas in 6 patients) and vascular malformations (venous malformation in 46 patients, lymphatic malformation in 57 patients and AV malformation in 62 patients) (Table 1).

Disease staging was carried out and showed that 210/245 patients were staged as having regional (extended) anomalies rather than local (localised) lesions. The anomaly volume was assessed using preoperative imaging (MRI, US). Seventy eight patients refused further conventional therapy (i.e. surgery) and elected to receive PDT, while 80 patients were not offered further conventional treatment and elected to receive PDT; the other 6 patients chose to undergo photodynamic therapy as the first intervention for their anomaly.

The patients' symptom-related disease included pain, bleeding, swelling, infection, disfigurement and breathing problems. One hundred eight patients were observed with chronic/recurrent pain which was associated with local pressure of the anomaly on nearby nerves. Forty six patients were observed bleeding which was mostly associated with trauma to their haemangiomas and AV malformations. Swelling problems were reported in 199 patients, while disfigurement was reported in 205 patients. Recurrent infection was identified in 63 patients, mostly in patients with lymphatic malformations. Breathing difficulties was associated with pathological growth in the nasal region in 5 patients. Since there is no validated assessment of quality of life tool for patients undergoing photodynamic therapy, our patients were asked only to report the nature of the complaint and not the severity (Table 2). No quality of life (QoL) questionnaires were applied in this study but merely patient's reports.

All patients were discussed at a multidisciplinary meeting involving surgeons, radiation and medical oncologists, interventional clinical radiologists and allied healthcare professionals. It was agreed to offer photodynamic therapy under general anesthesia, using 5-ALA as the photosensitising agent (200 mg $\mathbf{k g}$ intravenous for malformation, 500 $\mathrm{mg}$ as $20 \%$ ointment was administered on skin surface, $3 \%$ solution inject into vascular anomaly for 6 hours (Min. 6, Max. 24) prior to treatment). Treatment was carried out at a fluence of $10-20 \mathrm{~J} / \mathrm{cm}^{2}$ and a standardised fluence rate of $100 \mathrm{~mW} / \mathrm{cm}^{2}$ [14]. This would allow the agent to accumulate in the pathological area which would increase effectiveness. Patients were usually kept in a side room (with a dim light) to avoid systemic photosensitisation. Intraoperatively, an ultrasound probe was used to examine the centre and periphery of the anomaly when assessing volume, depth, invasion of large vessels, hollow organs and/or hard tissue. This was followed by insertion of 18 Guage $70 \mathrm{~mm}$ 
Citation: Rustamovich SR (2013) Results of the Treatment Vascular Anomalies with the 5 ALA Induced Photodynamic Therapy. Trop Med Surg 1: 157. doi:10.4172/2329-9088.1000157

\begin{tabular}{|c|c|}
\hline & No. $(\%)$ \\
\hline \multicolumn{2}{|l|}{ Gender } \\
\hline Male & $54(22)$ \\
\hline Female & $191(78)$ \\
\hline \multicolumn{2}{|l|}{ Age (years) } \\
\hline Mean & 1,2 \\
\hline Minimum-maximum & $0,1-34$ \\
\hline St. deviation & 0,4 \\
\hline \multicolumn{2}{|l|}{ Race } \\
\hline Central asian & $220(89,8)$ \\
\hline European & $19(7,7)$ \\
\hline Different & $6(2,4)$ \\
\hline \multicolumn{2}{|l|}{ Primary site } \\
\hline Oral cavity & $25(10,2)$ \\
\hline Upper face & $37(15,1)$ \\
\hline Middle face & $62(25.3)$ \\
\hline Lower face & $46(18.8)$ \\
\hline Neck & $38(15,5)$ \\
\hline Thorax, abdomen & $13(5,3)$ \\
\hline Upper extremity & $18(7.3)$ \\
\hline Lover extremity & $6(2.4)$ \\
\hline \multicolumn{2}{|l|}{ Vascular anomalies } \\
\hline \multicolumn{2}{|l|}{ Vascular tumours } \\
\hline Infantile haemangioma & $74(30.2)$ \\
\hline Congenital haemangioma & $6(2.4)$ \\
\hline \multicolumn{2}{|l|}{ Vascular malformations } \\
\hline Venous malformation & $46(18,8)$ \\
\hline Lymphatic malformation & $57(23.3)$ \\
\hline A-V malformation & $62(25,3)$ \\
\hline \multicolumn{2}{|l|}{ Staging } \\
\hline Local (localised) & $35(14,3)$ \\
\hline Regional (extended) & $210(85,7)$ \\
\hline \multicolumn{2}{|l|}{ Presentation } \\
\hline Disfigurement & $205(83,7)$ \\
\hline Swelling & $199(81,2)$ \\
\hline Pain & $108(44,1)$ \\
\hline Infection & $63(25,7)$ \\
\hline Breathing Problems & $5(2,0)$ \\
\hline Bleeding & $46(18.8)$ \\
\hline \multicolumn{2}{|l|}{ Previous management } \\
\hline Surgery & $78(31,8)$ \\
\hline Embolisation & $8(3,3)$ \\
\hline Sclerotherapy & $46(18.8)$ \\
\hline Alcohol therapy & $28(11.4)$ \\
\hline $\mathrm{CO} 2$ laser & $68(27,7)$ \\
\hline Adjuvant chemoradiotherapy & $11(4.5)$ \\
\hline None & $6(2,4)$ \\
\hline Further conventional treatment rejected & $165(67,3)$ \\
\hline Further treatment not offered & $80(32,7)$ \\
\hline \multicolumn{2}{|l|}{ Round(s) of PDT } \\
\hline 1 round & $39(15,9)$ \\
\hline 2 rounds & $13(5,3)$ \\
\hline 3 rounds & $159(64,9)$ \\
\hline 4 rounds & $11(4,5)$ \\
\hline 5 rounds & $23(9,4)$ \\
\hline \multicolumn{2}{|l|}{ Follow up (months) } \\
\hline Mean & 21 \\
\hline Minimum-maximum & $5-45$ \\
\hline St. deviation & 4,3 \\
\hline
\end{tabular}

Table 1: Demographics of the 245 Patients with Vascular Anomalies. long spinal needles under US-guidance into the target tissue. Great care was taken to ensure that the needles are inserted parallel to each other with 7-9 $\mathrm{mm}$ distance in between. If the treatment was close to a major blood vessel, a safety distance of $1 \mathrm{~cm}$ between the needle and the vessel was implemented.

An Iso-illumination treatment plan was carefully implemented and supervised by a senior physicist to ensure adequate light delivery to all suspect areas. A $635 \mathrm{~nm}$ diode laser was used for illumination. Diffuser fibres were used with/out bare polished tip fibres, with a core diameter of $400 \mu \mathrm{m}$, to deliver light. Light was then delivered from the fibres to the target tissue at $20 \mathrm{~J}$ per site. Tissues outside the target area were shielded completely to avoid photo activation by scattered or reflected light.

Pulse oximeters were repositioned at least every 30 minutes to avoid the risk of local skin burns and nail bed damage. All precautions were taken to avoid direct illumination of the patient with surgical lamps in theatres. Unplanned or emergency surgical interventions within 30 days from the photosensitiser administration were undertaken only if absolutely necessary and the potential benefits outweigh the risk to the patient. Precautions were applied to avoid exposure of skin and eyes to direct sunlight or bright indoor light during the first 15 days after injection. Patients were re $\neg$ introduced to normal light gradually. No drug interactions have been observed.

The patients were discharged from hospital care at a mean of 5 days (Min 3, $\max 9$ ) postoperatively. Patients were followed-up and asked to report on the outcome of their therapy if there is any improvement, no change or worsening of symptoms. Clinical assessment outcome was performed by a team of surgeons/physicians trained in photodynamic therapy at approximately 6 weeks postoperatively. The clinical assessment criterion was based on reduction of lesional size and improvement of the initial clinical symptoms. MRI imaging and US was performed 5-6 weeks postoperatively. Our radiological assessment parameters included: no response-progressive disease (increase in pathology size), no response-stable disease (no change in pathology size), minimal response (reduced size by $<25 \%$ ), moderate response (reduced size by $<50 \%$ ) and significant response (reduced size by 50 $75 \%)$. Identification of peri-lesional inflammation, assessing response to PDT was also reported. The time between the rounds was 3-6 months, with treatment indicated when the patient becomes symptomatic. The mean follow-up for those patients was 21 months (Min 5, Max 45).

\section{Statistical Analysis}

This was performed using the SPSS 17 (statistical package for social scientists) by an independent statistician. The patients' data were entered onto proformas which were validated and checked by interval sampling. The fields included a range of clinical, operative and radiological parameters related to the outcome following photodynamic therapy. The results were cross tabulated and the Chi-squared statistic was used to test for differences in the incidence of outcome. Fisher's exact test was used for the analysis of contingency tables and therefore to measure the P-value.

\section{Results}

Eighty five out of one hundred eight patients who presented longterm or recurrent pain reported significant improvement of their symptoms $(P<0.001)$; while the rest of the group reported no change. Also 43/46 patients with bleeding problems reported improvement $(P<0.001)$, and no patient was reported with worsening bleeding 
Citation: Rustamovich SR (2013) Results of the Treatment Vascular Anomalies with the 5 ALA Induced Photodynamic Therapy. Trop Med Surg 1: 157. doi:10.4172/2329-9088.1000157

Page 4 of 5

No. (\%)

No response-stable (no change in pathology size), no response-progressing (increase in pathology size), minimal response (reduced size by $<25 \%$ ), moderate response (reduced size by $<50 \%$ ) and significant response (reduced size by 50-75\%).

Patient's report

Pain problems

108

Improved $(P<0.001)$

$85(78,7)$

No change

$23(21,2)$

Worse

Bleeding problems

Improved $(P<0.001)$

$0(0.0)$

46

$43(93,5)$

No change

$3(6,5)$

Worse

$0(0,0)$

Swelling problems

199

Improved $(P<0.001) \quad 189(95,0)$

No change $10(5,0)$

Worse

Infection problems

Improved $(P<0.001)$

$0(0.0)$

No change

63

$61(96,8)$

$2(3,2)$

Worse

$0(0.0)$

Disfigurement

Improved $(P<0.001)$

205

$176(85,9)$

Worse $0(0.0)$

Breathing problems

Improved $(P<0.001)$

$(0.0)$

No change

5

$3(60,0)$

$2(40,0)$

Worse

Clinical assessment

No response

Minimal response

$0(0.0)$

Moderate response

Good response

Radiologic assesment

No response-stable

No response-progressing

$5(2,0)$

$22(9,0)$

$70(28,6)$

$148(60,4)$

Minimal response

Moderate response

Significant response

Complications

Pain

Bleeding

Swelling

Infection

Skin burn

Skin ulceration

Sensory nerve injury

Motor nerve injury

Hypopigmentation

Hyperpigmentation

$23(9.4)$

$5(2,0)$

$37(15,1)$

$78(31,8)$

$122(49,8)$

$12(4,9)$

$5(2,0)$

40 (16.3)

$2(0,8)$

$6(2,4)$

$0(0.0)$

$0(0.0)$

$0(0.0)$

$0(0)$

$6(2,4)$

Table 2: Patients Responses pre and Post-PDT, Clinical and Radiologica Assessments and Demographics.

episodes. Swelling improvement was observed in 189/199 patients and 176/205 patients reported improvement of their disfigurement; improvement in both parameters was significant $(P<0.001)$. Infection rate was reduced in $61 / 63$ patients who presented hemangioma and malformations $(P<0.001)$. Breathing improvement was evident in $3 / 5$ patients $(P<0.001)$ (Table 2$)$.
Clinical assessment showed that 148/245 patients had 'good response' to the treatment and $70 / 245$ had 'moderate response'. Radiological assessment, comparing imaging 6-week post-PDT to the baseline, showed stable pathology with no change in size in $23 / 245$ patients, minimal response $(<25 \%$ reduction) in $37 / 245$ patients, moderate response ( $<50 \%$ reduction) in $78 / 245$ patients and significant response (50-75\% reduction) in 122 patients. Five patients during the treatment showed no response with pathology progression (lymphangioma of the mid face) (Table 2, Figures 1 and 2).

Pain was reported by 12 patients and was mild-moderate. Bleeding was observed by 5 patients and no motor and sensory nerve injury was reported. Two patients reported postoperative infection and were treated with antimicrobials. No patient had skin ulceration/necrosis, skin burn was reported by 6 patients. Burn skin patients in sequence were observed hyper pigmentation (Table 2).

\section{Discussion}

Hemangioma prognosis established that haemangiomas resolve in $50 \%$ children by 7 of age. This case leads physicians to manage these patients in the conservative approach, but recent studies have showed that patients may present disease specific complication. This condition requires intervention and complete resolution in quite difficult to achieve. Problematic haemangiomas can cause severe functional issues including ulceration, disfigurement and affect breathing, swallowing and speech. Common locations for these problematic haemangiomas include face, ear, orbit, lower lip and airway [16].

Conventional management can include systemic or intra-lesional corticosteroids, beta blockers, chemotherapeutic agents, surgery, laser or a combination of these therapies. Where lesions are large, surgery can have marked adverse effect on form and function and due to difficulty in delineating, these lesions recurrences can be high. The side effects of chemotherapy are well known and radiotherapy carries the risk of inducing new tumour. Propranolol, a known non-selective beta-blocker, has been reported to induce regression in haemangiomas in newborns $[17,18]$. Sclerotherapy is the most common intervention in many centers around the world. Laser therapies have been reported as the most useful in the management of airway malformations and vesicular eruptions on mucosal surfaces. However, when it comes to complex lesions, surgery is always considered as the first option [19]. Resection of all diseased tissue is advocated as failure results in regrowth. Several studies have reported the use embolisation with varying substances (i.e. glue, coils and alcohol) in the management of AV malformations. This is mainly used in small and focal AV-malformations, but can be used in very large life-threatening AV- malformations 1 , followed by microvascular free flaps to reconstruct the defect [4].

\section{Photodynamic Therapy (PDT)}

Since PDT is a non-heat generating process, there is no bystander tissue heating, and connective tissues, such as collagen and elastin, are largely unaffected. As a result, many tissues heal with little scarring in comparison to thermal treatment and hollow organs such as trachea, the gastrointestinal tract and major blood vessels that maintain their mechanical integrity [20]. The effectiveness of PDT depends on the dosimetric profile. Ideally one would quantify the distribution of the light fluence rate, the optical properties, the drug concentration and tissue oxygenation for PDT. The other advantage of PDT is that it can be repeated without cumulative toxicity. The success of PDT is dependent on upon the depth of necrosis being greater than the depth of the individual malformation. Thus, interstitial PDT represents is ideal, 
Citation: Rustamovich SR (2013) Results of the Treatment Vascular Anomalies with the 5 ALA Induced Photodynamic Therapy. Trop Med Surg 1: 157. doi:10.4172/2329-9088.1000157

in that it allows more effective treatment of the target tissue volume $[17,20]$. This clinical study highlights the advantage of ultrasound, in combination with PDT. It is relatively simple to perform, easily available and examinations are non-invasive. It is inexpensive, quick, and convenient and there are no known harmful effects, since it uses non-ionising radiation suited to the soft tissues and it does not cause any pain. Ultrasound can be used to guide the optical fibres to the appropriate disease volume, enabling more accurate evaluation of treatment doses $[17,21]$.

The management of vascular anomalies continues to be extremely challenging. Although several modalities have been developed and the literature reports successful treatment in many, data from long term studies reports relapse in many and the need for re-treatment or another intervention. Photodynamic therapy is not superior to other modalities, but it is characterized by being one of the least invasive, being repeatable with no residual toxicity and with a minimal bystander effect on the overall tissue architect and integrity as well as nerves. The growing body of evidence regarding its efficacy, the increasing use of image guided PDT, and the innate minimally invasive characteristics of PDT suggest that it should become an important addition to the various techniques used in the management of vascular anomalies.

\section{References}

1. Drolet BA, Esterly NB, Frieden IJ (1999) Hemangiomas in children. N Engl J Med 341: 173-181

2. Jeong HS, Baek CH, Son YI, Kim TW, Lee BB, et al. (2006) Treatment for extracranial arteriovenous malformations of the head and neck. Acta Otolaryngol 126: 295-300.

3. Buckmiller LM, Richter GT, Suen JY (2010) Diagnosis and management of hemangiomas and vascular malformations of the head and neck. Oral Dis 16: 405-418.

4. Mulliken JB, Glowacki J (1982) Classification of pediatric vascular lesions. Plast Reconstr Surg 70: 120-121.

5. Wu JK, Bisdorff A, Gelbert F, Enjolras O, Burrows PE, et al. (2005) Auricular arteriovenous malformation: evaluation, management, and outcome. Plast Reconstr Surg 115: 985-995.

6. Perkins JA, Tempero RM, Hannibal MC, Manning SC (2007) Clinical outcomes in lymphocytopenic lymphatic malformation patients. Lymphat Res Biol 5: 169174.

7. Blei F (2008) Congenital lymphatic malformations. Ann N Y Acad Sci 113: 185194.
8. Boon LM, Mulliken JB, Enjolras O, Vikkula M (2004) Glomuvenous malformation (glomangioma) and venous malformation: distinct clinicopathologic and genetic entities. Arch Dermatol 140: 971-976.

9. Duyka LJ, Fan CY, Coviello-Malle JM, Buckmiller L, Suen JY (2009) Progesterone receptors identified in vascular malformations of the head and neck. Otolaryngol Head Neck Surg 141: 491-495.

10. Ethunandan M, Mellor TK (2006) Haemangiomas and vascular malformations of the maxillofacial region--a review. Br J Oral Maxillofac Surg 44: 263-272.

11. Schumacher M, Ernemann U, Berlis A, Weber J (2008) Treatment of venous malformations--comparison to lymphatic malformations. Lymphology 41: 139146.

12. de Visscher SA, Dijkstra PU, Tan IB, Roodenburg JL, Witjes MJ (2013) mTHPC mediated photodynamic therapy (PDT) of squamous cell carcinoma in the head and neck: a systematic review. Oral Oncol 49: 192-210.

13. Seccia A, Salgarello M, Farallo E, Falappa PG (1999) Combined radiological and surgical treatment of arteriovenous malformations of the head and neck. Ann Plast Surg 43: 359-366.

14. Sandell JL, Zhu TC (2011) A review of in-vivo optical properties of human tissues and its impact on PDT. J Biophotonics 4: 773-787.

15. Hennig G, Stepp H, Johansson A (2011) Photobleaching-based method to individualize irradiation time during interstitial 5-aminolevulinic acid photodynamic therapy. Photodiagnosis Photodyn Ther 8: 275-281.

16. North PE, Waner M, James CA, Mizeracki A, Frieden IJ, et al. (2001) Congenital nonprogressive hemangioma: a distinct clinicopathologic entity unlike infantile hemangioma. Arch Dermatol 137: 1607-1620.

17. Kohout MP, Hansen M, Pribaz JJ, Mulliken JB (1998) Arteriovenous malformations of the head and neck: natural history and management. Plast Reconstr Surg 102: 643-654.

18. Léauté-Labrèze $C$, Dumas de la Roque $E$, Hubiche T, Boralevi F, Thambo JB, et al. (2008) Propranolol for severe hemangiomas of infancy. N Engl J Med 358: $2649-2651$.

19. Mavrikakis I, Heran MK, White V Rootman J (2009) The role of thrombosis as a mechanism of exacerbation in venous and combined venous lymphatic vascular malformations of the orbit. Ophthalmology 116: 1216-1224.

20. Svanberg K, Bendsoe N, Axelsson J, Andersson-Engels S, Svanberg S (2010) Photodynamic therapy: superficial and interstitial illumination. J Biomed Opt 15: 041502.

21. Richter GT, Suen J, North PE, James CA, Waner M, et al. (2007) Arteriovenous malformations of the tongue: a spectrum of disease. Laryngoscope 117: 328335. 